\title{
The LSC glitch group: monitoring noise transients during the fifth LIGO science run
}

\author{
L Blackburn ${ }^{1}$, L Cadonati ${ }^{2}$, S Caride ${ }^{3}$, S Caudill $^{4}$, S Chatterji ${ }^{5}$, \\ N Christensen $^{3}$, J Dalrymple ${ }^{6}$, S Desai ${ }^{7}$, A Di Credico ${ }^{6}$, G Ely ${ }^{3}$, \\ J Garofoli ${ }^{8}$, L Goggin ${ }^{5}$, G González ${ }^{4}$, R Gouaty ${ }^{4}$, C Gray ${ }^{8}$, A Gretarsson ${ }^{9}$, \\ D Hoak ${ }^{10}$, T Isogai ${ }^{3}$, E Katsavounidis ${ }^{1}$, J Kissel ${ }^{4}$, S Klimenko ${ }^{11}$, \\ R A Mercer ${ }^{11}$, S Mohapatra ${ }^{2}, \mathbf{S}$ Mukherjee ${ }^{12}$, F Raab $^{8}$, K Riles $^{13}$, \\ P Saulson $^{6}$, R Schofield ${ }^{14}$, P Shawhan ${ }^{15}$, J Slutsky ${ }^{4}$, J R Smith ${ }^{6}$, R Stone ${ }^{12}$, \\ C Vorvick $^{8}$, M Zanolin ${ }^{9}$, N Zotov ${ }^{16}$ and J Zweizig ${ }^{5}$ \\ ${ }^{1}$ LIGO-Massachusetts Institute of Technology, Cambridge, MA 02139, USA \\ 2 University of Massachusetts, Amherst, MA 01003, USA \\ ${ }^{3}$ Carleton College, Northfield, MN 55057, USA \\ ${ }^{4}$ Louisiana State University, Baton Rouge, LA 70803, USA \\ ${ }^{5}$ LIGO_California Institute of Technology, Pasadena, CA 91125, USA \\ ${ }^{6}$ Syracuse University, Syracuse, NY 13244, USA \\ 7 The Pennsylvania State University, University Park, PA 16802, USA \\ ${ }^{8}$ LIGO Hanford Observatory, Richland, WA 99352, USA \\ ${ }^{9}$ Embry-Riddle Aeronautical University, Prescott, AZ 86301, USA \\ ${ }^{10}$ LIGO Livingston Observatory, Livingston, LA 70754, USA \\ ${ }^{11}$ University of Florida, Gainsville, FL 32611, USA \\ 12 The University of Texas, Brownsville, TX 78520, USA \\ 13 University of Michigan, Ann Arbor, MI 48109, USA \\ ${ }^{14}$ University of Oregon, Eugene, OR 97403, USA \\ 15 University of Maryland, College Park, MD 20742, USA \\ ${ }^{16}$ Louisiana Tech University, Ruston, LA 71272, USA \\ E-mail: desai@gravity.psu.edu
}

Received 4 April 2008, in final form 16 June 2008

Published 2 September 2008

Online at stacks.iop.org/CQG/25/184004

\begin{abstract}
The LIGO Scientific Collaboration (LSC) glitch group is part of the LIGO detector characterization effort. It consists of data analysts and detector experts who, during and after science runs, collaborate for a better understanding of noise transients in the detectors. Goals of the glitch group during the fifth LIGO science run (S5) included (1) offline assessment of the detector data quality, with focus on noise transients, (2) veto recommendations for astrophysical analysis and (3) feedback to the commissioning team on anomalies seen in gravitational wave and auxiliary data channels. Other activities included the study of auto-correlation of triggers from burst searches, stationarity of the detector noise and veto studies. The group identified causes for several noise transients that triggered false alarms in the gravitational wave searches;
\end{abstract}


the times of such transients were identified and vetoed from the data generating the LSC astrophysical results.

PACS numbers: $04.80 . \mathrm{Nn}, 95.55 . \mathrm{Ym}$

(Some figures in this article are in colour only in the electronic version)

\section{Introduction}

The 'glitch group' is one of the subgroups of the Detector Characterization Committee within the LIGO Scientific Collaboration (LSC). In this paper we shall use the term 'glitch' to denote any short-duration noise transient in the gravitational wave channel as well as transients in auxiliary channels. Glitches produced by environmental effects or instrumental malfunctions are a source of background for transient gravitational wave signals, such as those from unmodelled bursts or compact binary coalescences. Sufficiently strong glitches are also responsible for loss of lock and decreased observation time. The glitch group was established in 2003 to characterize noise transients in LIGO. At times these investigations revealed causes that could be fixed and those transients were eliminated. The group consists of members from the analysis groups searching for short duration gravitational waves from coalescing binary systems, supernovae or other astrophysical systems, as well as detector experts and operators from both the LIGO sites at Hanford and Livingston. There is substantial interaction between the glitch group and other detector characterization working groups such as Calibration, Data Quality, Dataset Reduction, Environmental Disturbances and Hardware Injections. This paper will focus on the activities and findings of the glitch group during the fifth LIGO science run ('S5') which started in November 2005 and ended in October 2007 [1]. In section 2, we describe the tools used by the group for the diagnoses of instrument artifacts. In section 4, we describe some of the findings that led to elimination of identified problems and in better data quality. Finally, we shall conclude by highlighting some of the post-S5 activities (section 5).

The glitch group provided a forum for experts in data analysis and detector commissioning to join forces, brainstorm and assess the performance of the LIGO detectors during the S5 run (4th November 2005-1st October 2007).

Goals of the glitch group during the fifth LIGO science run (S5) included (1) offline assessment of the detector data quality, with focus on noise transients, (2) veto recommendations for astrophysical analysis and (3) feedback to the commissioning team on anomalies seen in gravitational wave and auxiliary data channels.

The glitch group activities complemented realtime investigations and onsite detector troubleshooting, and provided guidance to the burst and compact binary coalescence (CBC) analysis groups in their veto choices.

Members of the glitch group conducted offsite shifts, each covering three-four days of data acquisition. Results from these shifts were discussed in weekly telephone conferences. Highlights from these shifts were also presented each week in the run coordination and detector characterization teleconferences. Detailed specialized investigations were carried out by individual glitch group members.

\section{Goals and methods}

Glitch group members analyzed LIGO data with several near real-time algorithms, with latency ranging from few minutes to a day. Some of these were run using the Data Monitoring Tool 


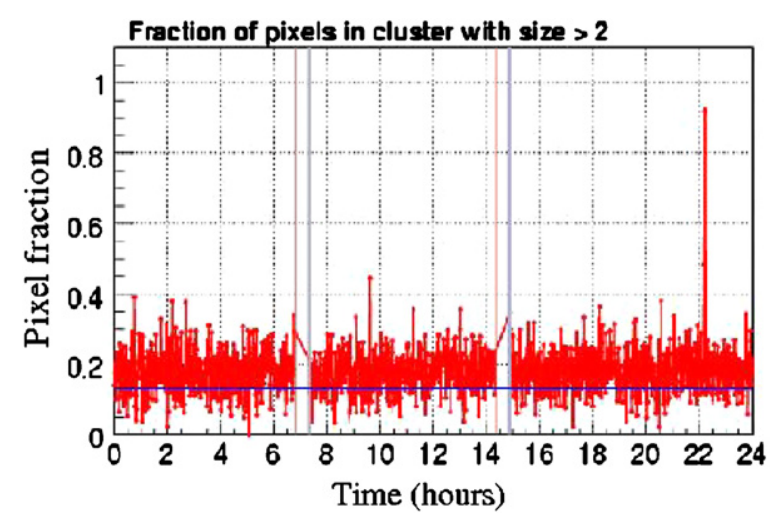

Figure 1. BurstMon [6] pixel fraction as a function of time (with one entry per minute). BurstMon first identifies the most significant $10 \%$ of time-frequency 'pixels' using a waveletbased decomposition of the data. The pixel fraction is defined as the fraction of these significant pixels that can be grouped into clusters of two or more pixels. Ideal noise typically produces isolated pixels resulting in a typical pixel fraction of 0.13 . Pixel fractions much greater than this value indicate a high rate of glitches in the data, which preferentially produce clusters of significant pixels.

(DMT) environment [3] within LIGO. The DMT is a set of algorithms that monitor various aspects of LIGO data quality, display status information and record data quality statistics. The goals of these near online algorithms ranged from searches for gravitational wave signals from unmodelled bursts and inspirals to studies of detector noise.

Block-Normal : Block-Normal is an algorithm designed to search for short-duration unmodelled gravitational wave bursts. It is based on a time domain analysis of the data and uses a Bayesian statistics figure of merit to select candidate events [4]. During a glitch shift, we scanned single interferometer outliers from Block-Normal using the event visualization tools.

BurstMon: BurstMon is the DMT tool for monitoring the burst detection performance of LIGO detectors. It is closely related to the Waveburst algorithm [5] that is used for untriggered gravitational wave burst searches using data from second, third and fourth LIGO science runs. This monitor produces three figures of merits: a measure of the rate of nonstationarity called 'pixel fraction', the real-time detector sensitivity to gravitational wave bursts and noise variability in various frequency bands. A sample plot from the 'pixel fraction' figure of merit is shown in figure 1. The term 'pixel' is used to denote a time-frequency bin and the pixel fraction is the fraction of pixels which can be grouped into clusters of two or more pixels. It indicates what fraction of the time-frequency volume is affected by the non-Gaussianity of the detector noise. For stationary Gaussian noise, its value is equal to 0.13. However for real LIGO data it could be as large as 1. More details on the Burstmon figures of merit are provided in [6].

InspiralMon: online searches for inspiralling binary compact objects were done using matched-filter-based searches for compact object mergers between 1 and $3 M_{\odot}$ using secondorder post-Newtonian stationary phase templates [8]. All separate triggers within a $15 \mathrm{~s}$ timewindow were clustered into one set. These triggers were not used for the actual gravitational wave search (which uses coincidence between detectors, a larger template bank and several signal-based vetoes), but were very useful for diagnostic purposes. The signal-to-noise ratio of the loudest trigger was displayed in the control room every minute. During the glitch 


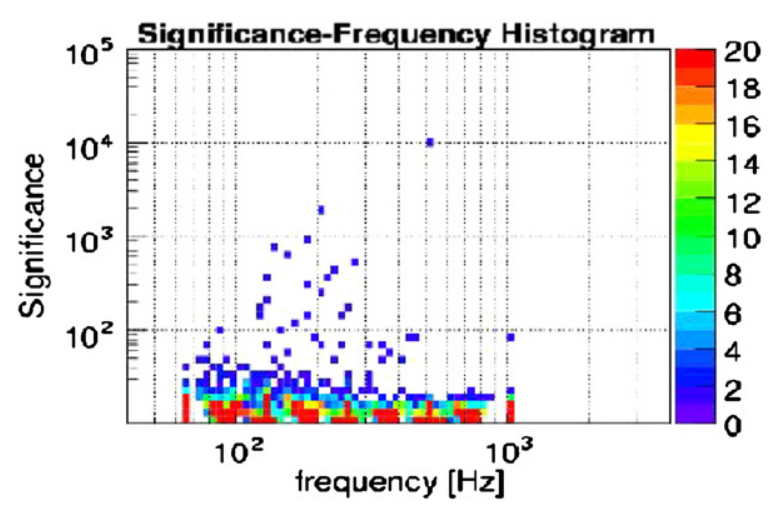

Figure 2. KleineWelle significance versus frequency (over a one-day integrated period). The color-scale is the number of events in a given significance-frequency bin.

shifts, we examined the loudest 20 single interferometer CBC triggers produced each day with signal-to-noise ratio greater than 15.

KleineWelle: KleineWelle [9] is a single interferometer event trigger generator. It is based on the dyadic wavelet decomposition of a time-series. The wavelet transform provides time-frequency localization of signal energy represented by the wavelet coefficients of the decomposition. During S5, KleineWelle analyzed in near-realtime (and offline) the gravitational wave channel and a variety of auxiliary channels for the three LIGO detectors and GEO. A variety of diagnostic plots were produced from these triggers. Multi-dimensional classification analysis was also done using these triggers [2]. Plots of the trigger rate for a given channel with low and high thresholds could be produced with a graphical web-based interface. During the glitch shifts, we explored both double-coincident (between the two LIGO Hanford interferometers) and triple-coincident $\mathrm{KleineWelle} \mathrm{triggers} \mathrm{using} \mathrm{event} \mathrm{visualization} \mathrm{tools.}$ We also examined various other diagnostic plots such as trigger auto-correlations, trigger periodicities, trigger significance as a function of frequency etc. Anomalous features in the auto-correlation plots are usually due to enhanced microseismic noise. Some of these plots are shown in figures 2 and 3.

NoiseFloorMon: NoiseFloorMon is a monitor to detect slow drifts in the noise floor [7]. It was applied to the gravitational wave channel and to various seismic channels. During glitch shifts we typically looked at minute trends of threshold crossings and cross-correlations with seismic channels.

QOnline: The QOnline pipeline is an online multi-resolution time-frequency search for statistically significant excess signal energy. It is equivalent to a templated matched filter search (in the whitened signal space), whose basis functions are sinusoidal Gaussians of varying central time, central frequency and the quality factor $Q$. Details on the Q-transform are provided in [10]. The algorithm was run online on data from the three LIGO detectors, VIRGO and GEO. During the glitch shifts, we examined the trigger trends from the QOnline pipeline (see figure 4) and a scan of the loudest event within each hour.

Besides the above near-online analysis, we also studied online figures of merit (which are usually produced in realtime in the control room) such as the effective distance to which LIGO is sensitive to binary neutron star inspirals, as well as environmental factors such as wind, band-limited seismic noise, etc. See figure 5 for a plot of band-limited microseismic noise usually looked at during these shifts. 


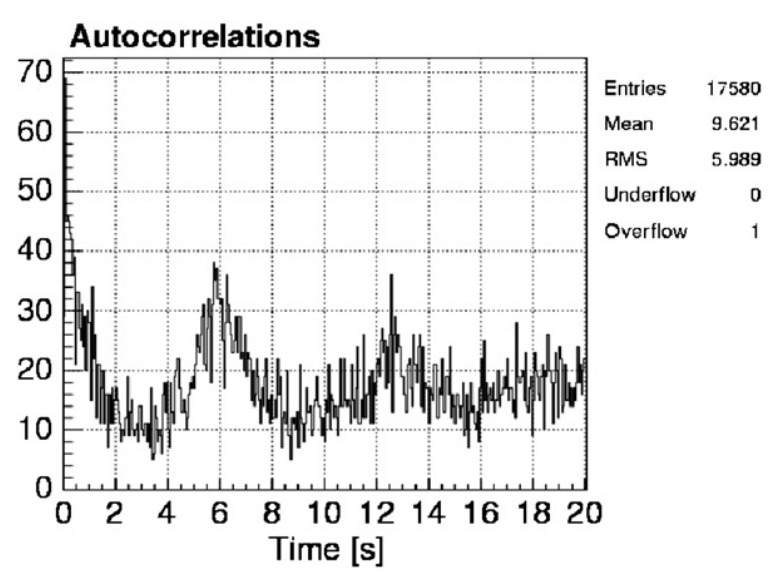

Figure 3. Auto-correlogram of the KleineWelle triggers. The auto-correlogram is a histogram of the time-difference between a given trigger and all other triggers. A peak in this plot is an indicator of periodicity which on a time-scale of a few seconds is due to enhanced microseismic noise.

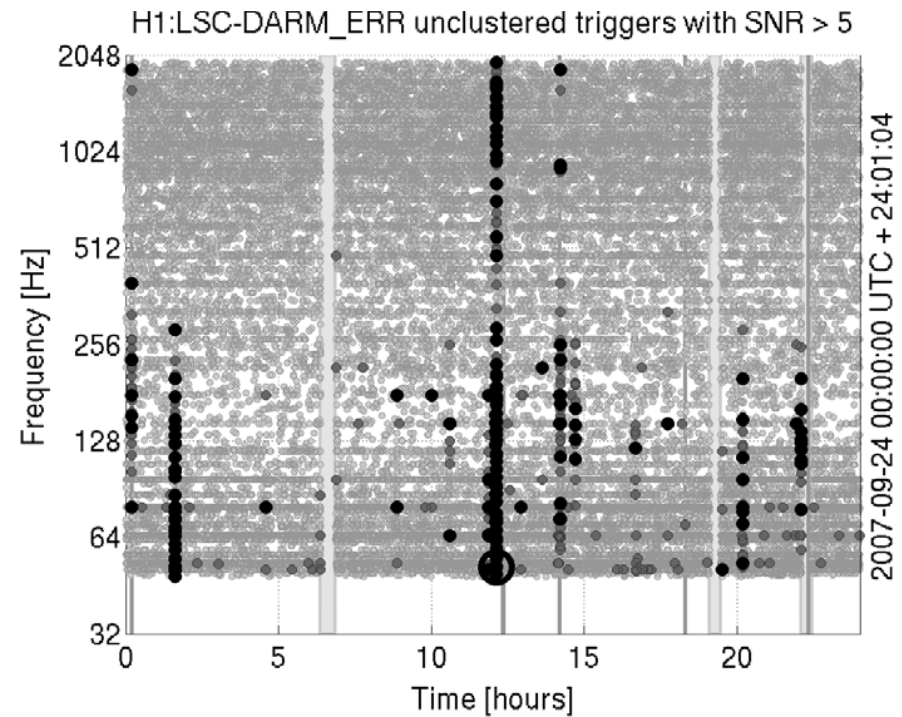

Figure 4. Time-frequency scatter plot of triggers from the QOnline analysis of the $4 \mathrm{~km} \mathrm{LIGO}$ Hanford detector. The amount of grayscale indicates ranges of SNR, with light gray indicating SNRs from 5 to 10 , dark gray from 10 to 20, and black greater than 20 .

We also gained understanding of glitch mechanisms by listening to the whitened versions of glitch waveforms through high-quality audio systems, taking advantage of the fact that our search is carried out at audio frequencies. A wide range of input disturbances lead to glitches with no discernible differences. In fact, each interferometer seems to have a characteristic glitch waveform, each a variation of a few-cycle oscillation near $100 \mathrm{~Hz}$. Further study is exploring the exceptions to this general rule, including longer-duration ('more musical') tones, broad-band glitches and echoes. It is hoped that these studies will give a clue about the glitch mechanism(s), still undiagnosed. 


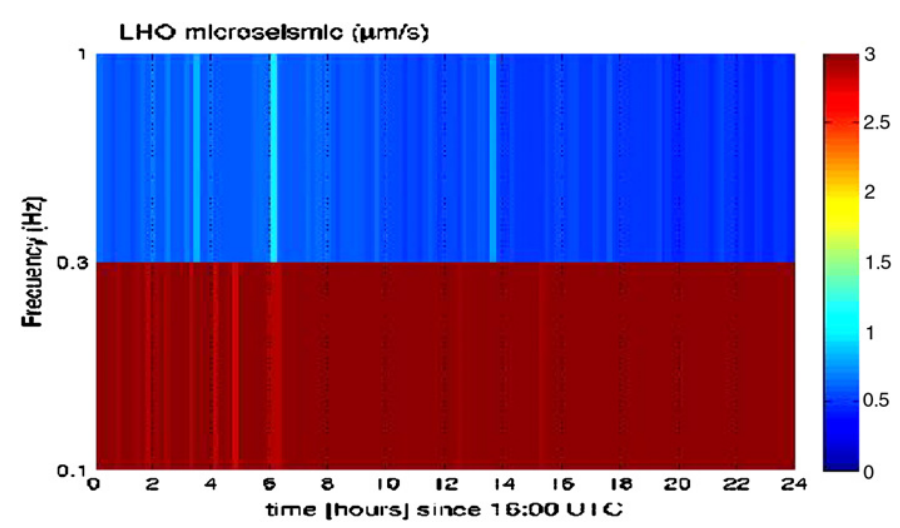

Figure 5. Band-limited seismic noise in the $0.1-1 \mathrm{~Hz}$ band at Hanford. Such high microseism could cause peaks in the auto-correlogram seen in figure 3.

\section{Event visualization tools}

We used two event visualization tools for a better insight into the behavior of detectors at any particular time of interest, which provide snapshots of the LIGO auxiliary and environmental channels as well as the gravitational wave channel. These are similar to event display tools routinely used in high energy physics experiments to depict the tracks of particles. Times of interest included outliers from the burst and $\mathrm{CBC}$ searches, hardware signal injections, gamma-ray-burst arrival times, environmental injections, etc. The tools provided insight into the behavior of the detectors at a given time and helped identify a few 'smoking gun' causes of loud glitches, data corruption and sources of lock-loss.

Event-Display: The Event-Display is also a web-based event visualization tool which shows the time-series and frequency spectrograms of a fixed set of channels along with various diagnostic information on the state of the detectors at that time, and output from the Parameter Estimation [11] code. The intensity in a given time-frequency bin is normalized by the median. One example of this type of specialized spectrogram of a glitch in the calibration channel is shown in figure 6.

QScan: QScan is used to investigate multiple detector channels around times of interest. QScan produces 'Q spectrogram' displays, based on the same transform used by the QOnline analysis. For statistically significant channels, QScan produces thumbnails of the time-series and ' $Q$ spectrograms' in three different time-windows $( \pm 0.5 \mathrm{~s}, \pm 2 \mathrm{~s}$ and $\pm 8 \mathrm{~s})$ on a webpage. The list of channels to look at can be defined with a configuration file. This tool has been extensively used in the control room by operators and science monitors to diagnose lock-losses and is also used to look at the various channels in the VIRGO detector. A QScan of a glitch in the voltmeter channel is shown in figure 7.

\section{Some results from the glitch group efforts}

During S5, the glitch group provided an offline forum to explore and discuss the day-to-day performance of the LIGO detectors, and provide commissioners with valuable feedback from a data analysis perspective. More importantly, these investigations led to the creation of several data quality flags which are used as vetoes in the analysis of S5 data. The identification of noise transients is particularly important for the development of vetoes for the burst and CBC searches. Similar work has been conducted by the LSC in the past [12-15]. For burst 


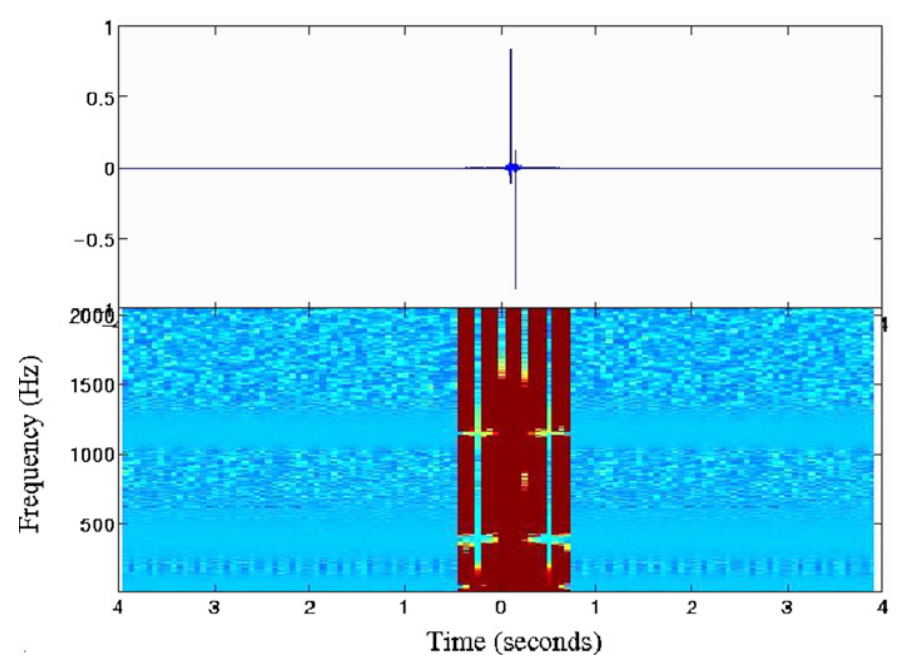

Figure 6. Superposed time-series and median-normalized spectrogram as it appears in the Event-Display of a glitch in the calibration channel, after the application of linear predictive error filter (defined in [10]).

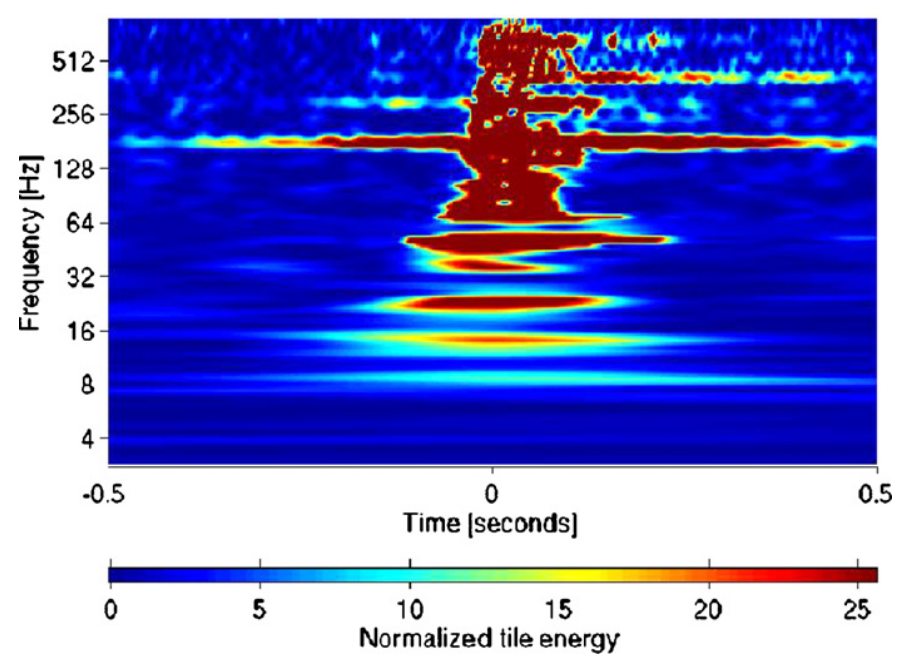

Figure 7. QScan of a glitch in a voltmeter channel, after data whitening with a linear predictive error filter (defined in [10]).

searches using the third LIGO science run the channel containing control signals from the power recycling cavity was used as a veto channel [15]. A discusson of various data quality flags and veto channels used for burst searches with data from the fourth LIGO science can be found in [16].

We provide a few examples of how our work helped commissioning efforts and improved data quality during the fifth LIGO science run. Although a detailed summary of all the investigations done by the glitch group over almost two years is beyond the scope of this paper, we list some of the most relevant results obtained: 


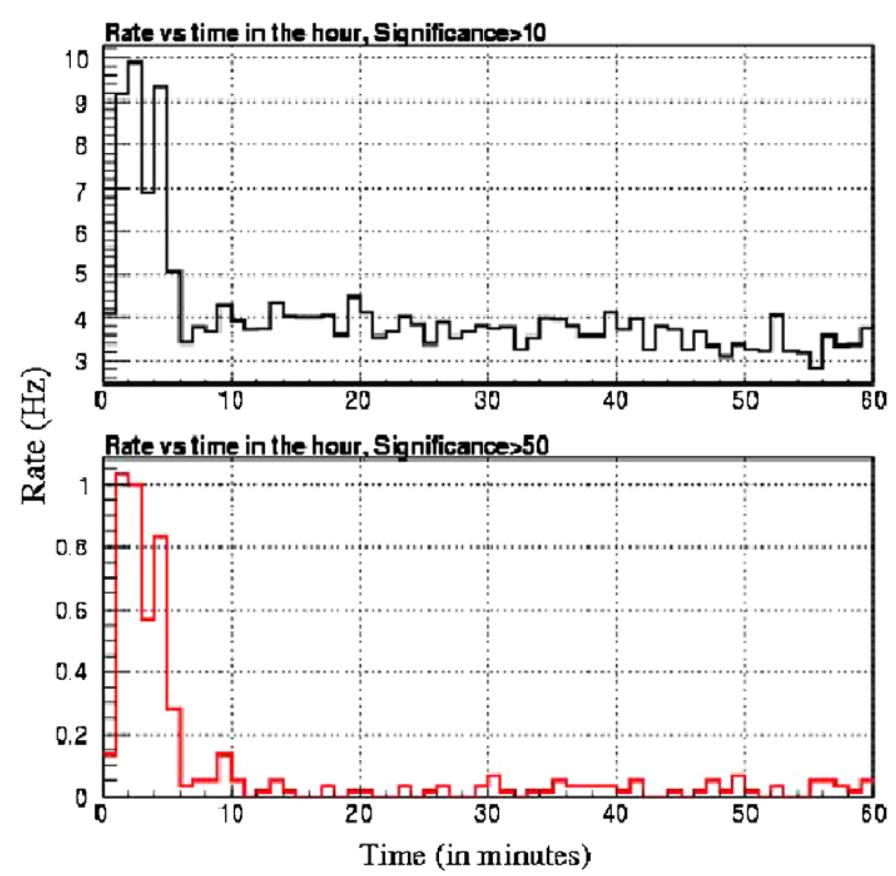

Figure 8. Histogram of the KleineWelle L1 event rate within an hour over a one day period when the detector experienced hourly glitches. The top and bottom panels show the rate of events with $\mathrm{KleineWelle} \mathrm{significance} \mathrm{greater} \mathrm{than} 10$ and 50 respectively.

- Near the start of S5, we were able to track down causes of lock loss in the Livingston interferometer due to 'channel hopping', when signals intended to drive the amplitude of an auxiliary laser were instead injected in a channel pushing the detector mirrors. These are similar to the example of the calibration glitch shown in figure 6 . The control and data system was fixed to monitor and prevent this artifact in the rest of the run.

- We found that many coincident $\mathrm{H} 1-\mathrm{H} 2$ glitches were also coincident with events in magnetometer and voltage channels (figure 7). Our investigation of several of these events revealed that they coincided with circuit breaker trips, shorts and other faults in high-voltage transmission lines that are connected to power substations near Hanford. The effect of these power grid events on the interferometer was consistent with what we expected from coupling of the ambient magnetic field transients to the permanent magnets on the test masses. The times of these and other power grid disruptions were flagged to prevent false alarms.

- Starting from October 2006, L1 experienced periodic glitches near the beginning of the hour, which were recognized as due to the digital snapshots of the various diagnostic information about the detector which happened once every hour. This was found by looking at the histograms of the KleineWelle event rate as a function of time within an hour as shown in figure 8.

- We tracked down the cause of a few outliers in the gravitational-wave channel to asymmetric response in the four photodiode signals used in the optical setup. Dedicated monitors were written following these findings to look for similar glitches caused by asymmetric photodiode response throughout S5. The glitches were believed to be due to dust along the beam path to the photo-diodes and was confirmed by specalized glitch injections in the post S5 period. 
- We have also done a classification of data quality flags into four categories with different levels of severity. These are classified into category 1 (which includes data that will not be analyzed), category 2 (where vetoes will be applied only in post-processing), category 3 (which are advisory flags used for detection confidence) and category 4 (which are advisory flags used to exert caution in case of a detection candidate). All these flags will be used for forthcoming burst and CBC papers using S5 data.

\section{Conclusions and future work}

Due to the long duration of S5 run, work is still in progress to wrap up all the S5 related glitch group efforts. The most important S5 related task still in progress is the creation of data quality flags and this is being done in collaboration with members from the Data Quality group. Another major effort is to follow-up possible coincident events from burst and CBC searches to assess the data quality at the time of the candidate and thus their statistical significance [17]. After S5, there were a few externally induced glitches and environmental injections. Some work has started using event visualization tools to characterize these glitches. We are also providing guidance to the Dataset Reduction group regarding choice of channels and sampling rates which need to be archived for the current Astrowatch program and future LIGO science runs. Thanks to the systematic effort of the glitch group, many artifacts were identified and the times were flagged, producing better data quality which allows for better astrophysical results, as well as improved confidence in any candidates that may be identified in the future. Many of the tools developed will be used in future runs for automated identification of the artifacts. The group effort has been very successful, and will likely continue and be improved in the future.

\section{Acknowledgments}

LIGO was constructed by the California Institute of Technology and Massachusetts Institute of Technology with funding from the National Science Foundation and operates under cooperative agreement PHY-0107417. We would like to thank the Bonneville Power Administration, particularly Mike Viles, for providing and interpreting records of power grid events. We are grateful to all members of the LIGO Scientific Collaboration for feedback and support for our work and especially to all the operators and members from the commissioning team who tirelessly worked for two long years to ensure a high duty cycle and good sensitivity during the fifth LIGO science run. This paper was assigned LIGO document number P080016.

\section{References}

[1] Abbott B et al (LIGO Scientific Collaboration) 2007 LIGO: The laser interferometer gravitational-wave observatory Preprint arXiv:0711.3041

[2] Mukherjee S (for the LIGO Scientific Collaboration) 2007 Class. Quantum Grav. 24 S701

[3] Zweizig J 1999 LIGO Technical Document G990079-35

[4] McNabb J et al 2004 Class. Quantum Grav. 21 S1705

[5] Klimenko S et al 2004 Class. Quantum Grav. 21 S1685

[6] Klimenko S 2005 LIGO Technical Document G050192-00-Z

[7] Mukherjee S 2006 Class. Quantum Grav. 20 S925

[8] LIGO Scientific Collaboration 2007 Technical Document T-070109-01-Z

[9] Chatterji S, Blackburn L, Martin G and Katsavounidis E 2004 Class. Quantum Grav. 21 S1809

[10] Chatterji S 2005 PhD Thesis MIT

[11] Sylvestre J 2002 Phys. Rev. D 66102004 
[12] Christensen N (for the LIGO Scientific Collaboration) 2005 Class. Quantum Grav. 22 S1059

[13] Christensen N, Shawhan P and Gonzalez G (for the LIGO Scientific Collaboration) 2004 Class. Quantum Grav. $20 \mathrm{~S} 1747$

[14] Cadonati L and Katsavounidis E (for the LIGO Scientific Collaboration's Bursts Working Group) 2003 Class. Quantum Grav. $20 \mathrm{~S} 633$

[15] Di Credico A (for the LIGO Scientific Collaboration) 2005 Class. Quantum Grav. 22 S1051

[16] Abbott B et al (LIGO Scientific Collaboration) 2007 Class. Quantum Grav. 245343

[17] Gouaty R (for the LIGO Scientific Collaboration) 2008 Preprint arXiv:0805.2412 